ROCZNIKI HUMANISTYCZNE

Tom LXIX, zeszyt $8-2021$

DOI: https://doi.org/10.18290/rh21698-7

\title{
KATARZYNA MANIOWSKA
}

\section{STUDIO COMPARATIVO \\ DELLE PAROLE COMPOSTE E POLIREMATICHE NEL LINGUAGGIO MEDICO ITALIANO E POLACCO}

\author{
A COMPARATIVE STUDY OF COMPLEX WORDS \\ IN POLISH AND ITALIAN MEDICAL TERMINOLOGY
}

Abstract

Dardano reminds that in Italian medical terminology "the Latin and Greek component constitutes a conspicuous patrimony" (Dardano 501, 514). The same can be said for Polish, although the Greco-Latin elements used in medical language are very often accompanied by their synonyms of Slavic origin. In Italian, such terms as chondromatosis, pharyngoplasty or nephropathy belong to both the technical and popular language, whereas in Polish there are terminological pairs: chondromatoza - chrzęstniakowatość, faryngoplastyka - plastyka gardła, nephropathy choroba nerek. They may be used according to the communicative situation and the register.

In Italian, in many cases it is possible to form propositional and adjectival phrases such as: ernia del disco/ernia discale, exeresi dei linfonodi/exeresi linfonodale. The construction with the complement, although admissible in certain cases, does not seem appropriate in the case of the higher register of the specialised language. The corresponding terms in Polish will have only one variant: przepuklina kręgostupa, usunięcie węzłów chtonnych.

A comparative study of complex words in Polish and Italian medical terminology will enable us to identify some differences in the two linguistic systems, and indicate their syntactic and semantic functions.

Key words: language for specific purpose; medicine; complex words.

Katarzyna Maniowska, PhD - Università Cattolica Giovanni Paolo II di Lublino, Facoltà di Scienze Umanistiche, Dipartimento di Linguistica; e-mail: katarzyna.maniowska@kul.pl. ORCID: https://orcid.org/0000-0002-8682-7951. 


\section{INTRODUZIONE}

Lo studio comparativo delle lingue è un compito affascinante poiché nella diversità, apparentemente inconciliabile, capita che si manifestino similitudini inaspettate.

L'analisi comparativa del linguaggio della medicina è ancor più attraente in quanto, grazie al raffronto di due sistemi linguistici utilizzati in un preciso ambito della scienza, si possono osservare fenomeni particolari. Si può assumere che la medicina più che ogni altra scienza include dei concetti universali. Al tempo stesso, essendo una scienza universale, la medicina costituisce un perfetto oggetto di studio comparativo in quanto sembra condizionata dalla cultura e dai fattori geo-storico-sociali che si traducono in varietà locali del linguaggio medico. Lo studio comparativo dei diversi livelli del linguaggio della medicina (sintattico - morfologico - lessicale) in diverse lingue permette di analizzare elementi intrinseci al linguaggio scientifico indipendentemente dalla lingua. D'altra parte, con lo studio comparativo in ambito medico si ottiene un ricco materiale di ricerca sulle caratteristiche di ogni lingua a prescindere dal contesto linguistico. Vale a dire, nello studio comparativo vengono messi sotto la lente di ingrandimento alcuni aspetti di un dato sistema linguistico che persistono a prescindere dal linguaggio medico.

Oltre alle considerazioni teoretiche, il presente studio intende offrire dei risvolti pratici a chi dovrà confrontare le due lingue nell'atto della traduzione. Nel processo di traduzione più che mai si manifesta quanto insidiosa possa essere la compresenza delle lingue, nonostante sia medesimo il contesto in cui esse vengono a trovarsi. Le parole di Maria Ibba, studiosa della lingua inglese, si possono benissimo riferire a qualunque lingua, inclusi l'italiano e il polacco medico: "L'inglese «scientifico» ha in comune con l'inglese «generico» il sistema, formale fonologico-grammaticale-sintattico, e i meccanismi retorici. La diversità sta nell'utilizzazione di certi aspetti particolari del «sistema lingua» a preferenza di altri" (Ibba 20).

Da una parte, le forme delle due lingue - oggetto dell'analisi - sono distinte, in quanto appartengono a due diverse famiglie linguistiche e dall'altra invece, appaiono pressoché uguali in quanto entrambe compaiono nel preciso contesto del linguaggio specialistico. Siccome i fenomeni designati sono gli stessi, è interessante vedere se siano anche uguali i meccanismi attuati a designarli. In principio sorge subito la domanda chiave: sarà la realtà a plasmare la lingua o viceversa? 
L'ambito di questa ricerca comparativa verrà ristretto alle parole composte e polirematiche per illustrare le particolarità proprie a due sistemi linguistici, per indicare punti comuni e soprattutto per ribadirne le differenze che costituiscono potenziali difficoltà traduttive.

\section{PAROLE COMPOSTE}

Per poter analizzare le parole composte in chiave comparativa risulterà utile ricordare la definizione del fenomeno inteso un po' diversamente nell'ambito della ricerca linguistica italiana e quella polacca. Grossmann e Rainer definiscono i composti italiani come "costruzioni complesse che si formano da parole che possono appartenere a categorie lessicali diverse oppure alla stessa categoria" (Grossmann, Rainer 33).

In italiano si dividono i composti in composti stretti i quali "hanno subito un amalgama fonologico" (Grossmann, Rainer 33), per esempio gentildonna (gentile + donna), e composti larghi "nei quali le parole costituenti mantengono la loro individualità fonologica e semantica" (Grossmann, Rainer 33), per esempio pescespada, cassaforte, capotreno.

I composti sono percepiti come parole formate in base agli elementi formativi iniziali o terminali definiti da alcuni prefissoidi e suffissoidi ${ }^{1}$ (Grossmann, Rainer 70-95, 99-116), oppure confissi, cioè "un elemento semantico pieno che, in quella forma e con il suo specifico significato, non ha in genere esistenza come parola autonoma" (De Mauro 68-69). A prescindere dalle problematiche terminologiche che derivano da tale distinzione, i composti neoclassici sono formati da elementi di origine greca o latina che possiedono un significato compiuto, tuttavia non accade che formino una parola indipendente. In polacco, il composto è classificato similmente ai composti italiani, cioè come costruzioni di almeno due diverse unità lessicali che formano un unico insieme accentato e grammaticale, inoltre esso viene declinato

\footnotetext{
${ }^{1}$ Sebbene la questione non costituisca oggetto della presente analisi, si segnala che la distinzione tra la coppia prefissi-suffissi e prefissoidi-suffissoidi non è netta ed è continuamente messa in dubbio: "La difficoltà di distinguere i prefissi dalle semiparole si manifesta nelle etichette che ricevono gli elementi non autonomi che compaiono in parole complesse quando non sono definiti prefissi: essi possono ricevere l'etichetta di «prefissoide», di «confisso», o più frequentemente di «primo elemento» (Montermini 26). In altre occasioni gli stessi elementi vengono denominati "elementi di forma colta" (Dardano), "elementi formativi scientifici" (Serianni), "elementi formativi scientifici e tecnici di origine greca o latina" (Tekavčić).
} 
come una parola omogenea ${ }^{2}$. Secondo un'altra definizione, le parole complesse vengono divise in composti propri, che grosso modo corrisponderebbero ai composti larghi in italiano, in cui il composto neoformato richiede un affisso o una desinenza che agglutina gli elementi, mentre un altro tipo di composti, definiti in polacco come zrosty, unisce indistintamente tutte le sue parti ${ }^{3}$, il che fa pensare ai composti stretti in italiano. A livello di definizioni si può notare che già la tipologia di composti in polacco è di duplice natura, la quale è principalmente determinata dalla presenza o meno di declinazione nel processo di formazione delle parole.

Un punto comune dei composti italiani e polacchi consiste nel fatto che tali parole, una volta formate, compongono un'unità inscindibile che denota un fenomeno solo. Nell'ambito del presente studio, la nostra attenzione si sposterà sulla questione di composti neoclassici in quanto sono loro a prevalere nella terminologia medica. A tale proposito si può dire che essi "presentano forti analogie con i composti di forme libere: sono composti di due elementi dotati di significato e, soprattutto, sono composti di elementi che, se non sono liberi, sono riconoscibili" (Arcodia 37). L'elemento neoclassico, a differenza degli affissi derivazionali, possiede un significato completo, nonché "funge da costituente «pieno», da radice" (Arcodia 37).

La terminologia medica è particolarmente duttile nel caso della formazione di nuove parole, proprio grazie a questo principale ingrediente neoclassico che offre la possibilità di combinazioni pressoché illimitate:

(...) l'esistenza in italiano, e in generale nelle lingue d'Europa, soprattutto indoeuropee, di una classe di semiparole è dovuta quasi esclusivamente a ragioni storiche e sociali,

\footnotetext{
${ }^{2}$ La parola complessa (złożenie) viene definita come una parola costituita dall'unione di due diverse unità lessicali le quali costituiscono un unico insieme accentato e grammaticale, ossia viene declinato come una parola sola, per esempio: żółtodziób, wiatronóg, rybołów (Głowiński 637).

${ }^{3}$ „Przez wyrazy złożone rozumie się strukturę motywowaną o dwóch (lub więcej) tematach słowotwórczych. Wyrazy złożone dzieli się na złożenia właściwe (potocznie: złożenia) i zrosty. Złożenia właściwe charakteryzują się brakiem fleksyjnych wykładników relacji syntaktycznych członów oraz obecnością formantu, np. dwutygodnik, wiercipięta. [...] Zrosty charakteryzują się tym, że nie tracą elementów fleksyjnych oraz tym, że nie mają interfiksów i formantów afiksalnych, np. herod-baba, mysikrólik" (Grochola-Szczepanek 61). [Per parole composte si intende una struttura con due (o più) radici formativi. Le parole composte vengono suddivise in composti propri (correntemente: zlożenia) e zrosty. I composti propri si caratterizzano per assenza di elementi flessionali delle relazioni sintattiche delle singole parti e dalla presenza di elementi formanti, ad esempio dwutygodnik, wiercipięta. [...] i composti di tipo zrosty si distinguono per il fatto di non perdere elementi flessionali e di non aver né infissi né altri elementi di tipo affissale, per esempio: herod-baba, mysikrólik; ove non indicato diversamente i frammenti citati sono stati tradotti dell'autrice].
} 
assolutamente indipendente dalla loro struttura. La principale di esse è il prestigio che è stato accordato per secoli al latino e al greco antico nella civiltà europea, e il fatto che tali lingue hanno fornito (e continuano a fornire) la base per numerosissime creazioni nel linguaggio tecnico-scientifico" (Montermini 31).

Non è escluso che la similitudine nella formazione delle parole composte dotte non ammetta anche delle discordanze. Per facilità le raggrupperemo in tre categorie maggiori, giustapponendo i termini italiani e polacchi, prendendo come spunto la base neoclassica, l'assenza o la presenza della quale diventa un tratto distintivo nel caso della terminologia polacca.

\subsection{ELEMENTI NEOCLASSICI: IL CASO DELLA BASE GRECO-LATINA}

Al primo gruppo appartengono termini che appena lievemente modificati ricalcano la stessa forma del termine neoclassico. I termini scientifici neoclassici vengono integrati nel sistema linguistico come prestiti, nonostante conviva il meccanismo di formazione di termini binari, di cui si parlerà più avanti:

W grupie ścisłych terminów greckiego pochodzenia, które nie miały polskich równoznaczników, znalazły się: anamneza, ankiloza, kefalometria, diateza, epidemiolog, epikryza, flebolit, hematuria, kifoza, miastenia oraz metempsychoza. W parach dubletowych, gdy między wyrazem rodzimym a zapożyczeniem greckim zachodzily niewielkie różnice znaczeniowe, zachowywały się oba człony dubletu, które nazywały odpowiednie desygnaty. Oto przykłady: astenia (bezsilność), ataksja (bezład, nieład, bezładność), atonia (zwiotczenie), atrofia (zanik) oraz dendryczny (drzewiasty) ${ }^{4}$. (Kupidura 206)

Pare che il prestigio secolare, di cui gode tuttora il latino e il greco, possa rendere più agevole il compito di traduzione dei termini neoformati, i quali prevalentemente attraverso l'inglese, permeano altri sistemi linguistici. A differenza però dell'italiano, la comprensione dei termini dotti non avviene in polacco con la stessa immediatezza che in italiano, sia per la natura stessa del polacco molto più lontano dal latino e dal greco di quanto non lo sia l'italiano, sia anche per una certa esclusività dei termini neoclassici in polacco.

\footnotetext{
${ }^{4}$ Il gruppo di termini stretti di origine greca che non avevano equivalenti polacchi includeva: anamnesi, anchillosi, cefalometria, diatesi, epidemiologo, epicrisi, flebolite, ematuria, cifosi, miastenia e metempsicosi. Nelle coppie di termini duplici, quando c'erano lievi differenze di significato tra la parola nativa e il prestito greco, si conservavano entrambi gli elementi della copia duplice, che richiamavano i referenti appropriati. Alcuni esempi: astenia (impotenza), atassia (disturbo, inerzia), atonia (rilassamento), atrofia (riduzione) e dendritico (arboreo).
} 
Alcuni slavisti hanno notato che nel caso delle lingue slave, le parole composte invece sono considerate elementi estranei al sistema stesso di queste lingue, formato prevalentemente sull'esempio di prestiti, e quindi di modelli allogeni (Handke 5). È ancora più giustificato tener presente quest'affermazione nel caso dei composti medici, per eccellenza facenti parte del linguaggio internazionale, e solo in un secondo momento addottati da sistemi linguistici per così dire locali. Nel loro caso si nota con maggiore chiarezza l'uniformizzazione del linguaggio medico, in quanto la loro forma grafica di singoli lemmi muta discretamente:

Tab. 1. Raffronto di termini medici italiani e polacchi su base neoclassica

\begin{tabular}{|c|c|}
\hline termini medici italiani & termini medici polacchi \\
\hline angioplastica & angioplastyka \\
\hline biopsia & biopsja \\
\hline cifosi & kifoza \\
\hline coronarografia & koronarografia \\
\hline elettroforesi & elektroforeza \\
\hline emoconcentrazione & hemokoncentracja \\
\hline etiologia & etiologia \\
\hline istiocitosi & histiocytoza \\
\hline paranoia & paranoia \\
\hline radiografia & radiografia \\
\hline scoliosi & skolioza \\
\hline termometro & termometr \\
\hline tripsina & trypsyna \\
\hline tomografia & tomografia \\
\hline valinomicina & walinomycyna \\
\hline
\end{tabular}


Il linguaggio medico sembra essere un superstrato linguistico con le proprie regole che si insinuano all'interno dei sistemi linguistici polacco e italiano. Il potere di questo superstrato pare alquanto strabiliante, se si considera il fatto che a differenza delle lingue naturali è tenuto in vita in maniera pressoché artificiosa, grazie all'incessante dosaggio di termini specialistici coniati alla stessa velocità del progresso scientifico.

Per lo più, questa straordinaria ricchezza e vivacità lessicale neoclassica continua a fiorire anche a prescindere dagli aspetti morfologico-sintattici delle lingue classiche, sempre più amalgamata con prefissi e suffissi, abbreviazioni e sigle inglesi, per esempio: apudoma, arbovirus, bartonellosi, viomicina (Zieliński 73, 75, 97, 584).

Le differenze tra i due sistemi linguistici iniziano a manifestarsi quando da parole composte "internazionali" vengono formati altri termini per derivazione, come per esempio degli aggettivi:

“All'esame coronarografico non è possibile identificare l'ostio coronarico di sinistra" (Laghi, Rengo 36).

"Se il tumore all'esame bioptico si rivela a crescita lenta ci si limita cioè a eseguire esami periodici più ravvicinati” (Lalla 65).

"La curvatura senza rotazione è definita atteggiamento scoliotico e non necessità di alcun trattamento in quanto è di spontanea risoluzione" (Cabitza 94).
Podczas koronarografii nie można zidentyfikować lewego ujścia wieńcowego.

Jeśli w czasie biopsji guz okaże się wolno rosnący, należy ograniczyć się jedynie do przeprowadzenia dokładniejszych okresowych badań.

Skrzywienie bez rotacji jest definiowane jako postawa skoliotyczna i nie wymaga żadnego leczenia, ponieważ ustępuje samoistnie.

Infatti, in alcuni casi (esame bioptico, esame coronarografico), i termini neoformati per derivazione possono essere annoverati tra i cosiddetti tecnicismi collaterali legati "a esigenze di registro stilistico non a necessità denotative" (Serianni 128). Nel caso dell'italiano, il processo di formazione di termini scientifici continua però anche laddove l'invenzione di termini nuovi pare superflua:

L'esigenza di astrazione (...) porta al forte sviluppo degli aggettivi di relazione; caratteristico il sintagma di nome generico + aggettivo di relazione portatore dell'informazione effettiva in luogo di un sostantivo specifico: stato carenziale (carenza), riscontro autoptico (autopsia), esame bioptico (biopsia), età puberale (pubertà), fenomeni morbosi (malattie o patologie). (Grossmann 587). 
Per converso il polacco come lingua di arrivo non sempre potrà tollerare una certa sovrabbondanza concettuale dei termini che normalmente si presentano in una forma molto più semplificata. E viceversa, tradurre dal polacco un tecnicismo nella loro versione meno ricercata (necroscopia invece di esame necroscopico, mammografia invece di indagine mammografica, ascesso invece di raccolta ascessuale) potrebbe passare per un atteggiamento irrispettoso verso le regole stilistiche non scritte del linguaggio scientifico italiano. Attenzione però ad applicare questa regola indistintamente. Per esempio il termine formazione nodulare $e^{5}$ non è solo un addobbo stilistico del termine nodulo ${ }^{6}$. Parimenti l'atteggiamento scoliotico è un tecnicismo vero e proprio e trattare la sua forma come un'alternativa del termine scoliosi, come nel caso dei termini precedenti, sarebbe un grave errore.

La similitudine terminologica nel caso delle parole complesse italiane e polacche con la comune origine neoclassica potrà quindi risultare deviante nel momento in cui le leggi sintattico-morfologiche e stilistiche di ciascuna lingua si sovrapporranno a quelle lessicali.

\subsection{ELEMENTI NEOCLASSICI: CASO DELLA BASE SLAVA}

Uno dei tratti distintivi del lessico scientifico polacco consiste nella sostituzione di parole dotte di origine greco-latina con i loro equivalenti di origine slava. Alcuni studiosi del polacco spiegano tale fenomeno con ragioni storicopolitiche (Kupidura 201) vigenti nell'epoca in cui si cristallizzava il moderno linguaggio scientifico. Qualunque fossero le cause di tali scelte lessicali, il fatto è che al giorno d'oggi, una gran parte della terminologia scientifica, e quella medica in particolare, si oppone a forme lessicali internazionali.

\footnotetext{
${ }^{5}$ Il termine viene tradotto in polacco come zmiana węzlowa. In entrambe le lingue il termine viene usato per descrivere un'anomalia.

${ }^{6}$ In polacco węzet. Il termine può riferirsi sia a fenomeni fisiologici (nodo linfatico, nodo linfoide) che patologici, per esempio nodi di Brenner, nodi juxta-articolari.
} 
Tab. 2. Raffronto di termini medici italiani e polacchi su base slava

\begin{tabular}{|c|c|}
\hline termini medici italiani & termini medici polacchi \\
\hline angioma & naczyniak \\
\hline angiomiofibroma & naczyniakomięśniakowłókniak \\
\hline aponeurosi & rozcięgno \\
\hline diaframma & przepona \\
\hline ematoma & krwiak \\
\hline emotorace & krwiak opłucnej \\
\hline epidermofitosi & grzybica naskórkowa \\
\hline epidermomicosi & grzybica naskórka \\
\hline extrauterino & pozamaciczny \\
\hline glucosuria & cukromocz \\
\hline lipoma & tłuszczak \\
\hline liponefrosi & nerczyca thuszczowata \\
\hline mesotelioma & międzybłoniak \\
\hline metrorragia & krwotok maciczny \\
\hline periostio & okostna \\
\hline peritoneo & otrzewna \\
\hline tendinite & zapalenie ścięgna \\
\hline
\end{tabular}

Come si può notare, non di rado capita che le parole complesse italiane in polacco diventino unità polirematiche. Una parola complessa o un'unità polirematica in italiano non è necessariamente tale in polacco e viceversa. D'altronde succede anche il contrario, ossia composti medici polacchi in italiano troveranno i loro equivalenti sotto forma di locuzioni polirematiche, per esempio: colonna vertebrale - kręgoshup, donatore di sangue - krwiodawca. Tuttora, non sono state chiarite le ragioni per le quali alcuni termini vengano introdotti nel linguaggio scientifico sotto forma di presiti integrati e quelli non integrati. Una delle possibili spiegazioni è quella di economia del linguaggio, la quale 
predilige forme più immediate e compatte: "Niektóre pożyczki upowszechniły się w języku prawdopodobnie ze względu na kryterium ekonomiczne - formy obce były jednowyrazowe, polskie zaś stanowiły konstrukcje analitycznej (por. dypsomania/opilstwo przerywane" (Kupidura 206) ${ }^{7}$.

Un altro possibile motivo per una maggiore presenza di prestiti integrati è dovuto al fatto che i professionisti del settore saranno maggiormente esposti al linguaggio scientifico internazionale, per cui adottare un termine neoclassico nella sua forma appena lievemente modificata è il modo più immediato per introdurlo nel vocabolario.

A questo punto occorre segnalare che non sempre prevale la legge dell'economia del linguaggio, in quanto capita che si prediligano termini più complessi, mentre vengano scartate scelte probabili, per esempio:

bradifrasia > spowolniona szybkość mowy, anziché *bradyfrazja,

tachifrasia > przyspieszona szybkość mowy, anziché *tachyfrazja.

Tale meccanismo è ancora più singolare se si considera il fatto che nella formazione di termini riportati sopra si potrebbe aspettare che per analogia verrà applicato lo stesso meccanismo già attuato nella formazione di termini affini, per esempio: bradicardia > rzadkoskurcz/bradykardia; tachicardia > częstoskurcz/tachykardia. In altre parole, la massiccia presenza di prefissi e prefissoidi, nonché di suffissi e suffissoidi neoclassici non significa che gli stessi elementi formativi verranno usati secondo lo schema già esistente. Ciò contribuisce a una maggiore disomogeneità terminologica che potrà risultare insidiosa in quanto non sempre sono chiare le leggi di formazione dei termini scientifici.

Dal punto di vista del traduttore, infatti, il fenomeno della doppia natura della terminologia scientifica polacca risulterà ancor più insidiosa in quanto ad essa si aggiunge l'abitudine del traduttore a pensare in più lingue e conseguentemente alla sua inclinazione a optare per termini più facilmente accessibili, cioè per quelli che variano appena lievemente dal termine originale.

È perciò utile ricordare a chi è avvezzo al linguaggio scientifico internazionale di non seguire l'orma dei modelli linguistici dominanti in altre lingue, nello specifico in italiano e in inglese. Un'avvertenza nel caso di questi composti è quella di mantenere l'altissima attenzione per non ricadere in

\footnotetext{
${ }^{7}$ Alcuni prestiti si sono diffusi nella lingua probabilmente favoriti dal criterio economico: le forme straniere erano una parola sola, mentre quelle polacche erano un costrutto analitico (vedi: dipsomania/ubriachezza intermittente).
} 
errore terminologico che consisterà nell'uso di termini dal significato diverso dal termine originale, o peggio ancora, nella coniazione di termini specialistici probabili ma inesistenti:

[...] i traduttori devono prestare particolare attenzione ai falsi amici (vale a dire elementi lessicali che sembrano corrispondere a livello semantico a parole di un'altra lingua sulla base di una somiglianza fono/grafo/morfologica, ma che in realtà hanno un significato differente) oppure ai falsi amici parziali, casi in cui la parola può essere tradotta in modo equivalente e trasparente in certe occasioni ma non in altre. (Canepari 216)

Il traduttore, essendo esposto a una quantità amplissima di termini in entrambe le lingue e spesso anche nelle lingue ponte utili per approfondire l'argomento discusso nella traduzione, tenderà a vedere con molta più facilità le possibilità di formare parole nuove, il che non vuol dire affatto che tale termine circoli negli ambienti di specialisti. Tale tentazione è tanto più giustificata, se si prende in considerazione la terza categoria dei composti medici, in cui in maniera interscambiabile compaiono termini di origine neoclassica e slava.

\subsection{ELEMENTI NEOCLASSICI: CASO DELLA BASE SLAVA + GRECA E LATINA}

All'ultima categoria importante dal punto di vista delle divergenze esistenti tra la terminologia italiana e polacca abbiamo classificato i termini con la duplice forma. Esistono cioè termini medici che amalgamano le due categorie precedenti, ossia termini originatisi sulla base neoclassica e quella slava, come illustrano gli esempi nella seguente tabella:

Tab. 3. Raffronto di termini medici italiani e polacchi su base neoclassica e slava

\begin{tabular}{|c|c|c|}
\hline termine italiano & $\begin{array}{c}\text { termine polacco su base } \\
\text { neoclassica }\end{array}$ & termine polacco su base slava \\
\hline antimalarico & przeciwzimniczy & przeciwmalaryczny \\
\hline broncoesofagoscopia & bronchoezofagoskopia & $\begin{array}{c}\text { wziernikowanie oskrzeli i } \\
\text { przełyku }\end{array}$ \\
\hline bradicardia & bradykardia & rzadkoskurcz \\
\hline bradicinesia & bradykinezja & spowolnienie ruchowe \\
\hline bradilalia & bradylalia & spowolnione tempo mowy \\
\hline condroblasti & chondroblasty & \multicolumn{2}{c|}{} \\
\hline
\end{tabular}




\begin{tabular}{|c|c|c|}
\hline emodiluzione & hemodylucja & rozcieńczenie krwi \\
\hline eritropoiesi & erytropoeza & wytwarzanie krwinek czerwonych \\
\hline ettasia & ektazja & rozstrzeń, rozdęcie \\
\hline glicolisi & glikoliza & rozkład glukozy \\
\hline infiltrazione & infiltracja & nacieczenie, naciek \\
\hline irsutismo & hirsutyzm & nadmierne owłosienie \\
\hline
\end{tabular}

È importante ribadire il fatto che non si tratta di forme di termini interscambiabili, anzi più spesso la scelta lessicale è dettata dal registro e dal grado di specializzazione del testo in cui essi compaiono. Nell'uso delle coppie di tali termini si delinea una chiara distinzione a seconda della situazione comunicativa, vi si possono cioè indicare varietà diafasiche e diastratiche (cfr. Berruto 139-168):

Część wyrazów nieakceptowanych przez XIX-wieczną normę językową utrwaliła się we współczesnej polszczyźnie. Dotyczy to przede wszystkim terminów medycznych. Zgodnie z orzeczeniami normatywnymi z przełomu XIX i XX wieku przejęte z laciny formy funkcjonują jako profesjonalizmy medyczne, natomiast ich rodzimych odpowiedników używa się w mówionej odmianie języka polskiego ${ }^{8}$. (Kupidura 201)

In genere, i termini medici di origine slava compaiono nel linguaggio scritto e orale destinato al largo pubblico dei non professionisti, mentre i termini neoclassici con maggiore probabilità compariranno nei testi scritti destinati al ristretto gruppo di specialisti: "Specjalistyczne terminy łacińskie utrwaliły się w polszczyźnie ze względu na wąskie znaczenia, natomiast ich polskie odpowiedniki o szerszym znaczeniu przeszły na obrzeża języka lub zmieniły zakres użycia"9 (Kupidura 201).

I termini specialistici polacchi, in grado forse maggiore rispetto al linguaggio medico italiano, sono molto più dipendenti dalla situazione comunicativa, per cui il traduttore deve considerarli in primis come variante diafasica,

8 "Alcune parole non accettate dalla norma linguistica ottocentesca si sono affermate nel polacco contemporaneo. Questo vale principalmente per i termini medici. Secondo le decisioni normative definite a cavallo tra il XIX e il XX secolo, le forme prese dal latino funzionano come tecnicismi medici, mentre le versioni native vengono utilizzate nella forma parlata della lingua polacca".

${ }^{9}$ I termini latini specialistici si affermarono in polacco grazie al loro significato ristretto, mentre le loro controparti polacche con un significato più ampio passarono alla periferia della lingua o cambiarono l'ambito di utilizzo. 
"dipendente non dalla materia in sé, bensì dalla specifica situazione e funzione comunicativa" (Garzone, Santulli 27). Come affermano gli studiosi del fenomeno, "la variazione diafasica, legata alla situazione comunicativa, è resa particolarmente complessa, sia dal punto di vista teorico che descrittivo, dalla pluralità di fattori ad essa pertinenti e dal loro sovrapporsi negli usi concreti" (Holtus, Metzeltin, Schmitt 768). Sarebbe una banalità ripetere che nell'atto di tradurre, oltre che alla lingua, il traduttore deve badare alla situazione comunicativa, tuttavia l'argomento più ostico è appunto l'abilità del traduttore di cogliere regole quasi mai scritte dell'uso dei termini. Questa capacità va al di là di una mera abilità linguistica e cognitiva per le quali si distingue il professionista del settore di traduzioni specialistiche. Per una corretta scelta dei termini appropriati occorre valutare alcune invariabili del termine, tra cui il grado di intelligibilità e la complessità della forma e dei suoi derivati. Se contempleremo questi dati nel contesto più vasto del testo, con molta probabilità la traduzione del senso del termine potrà approssimarsi a quello originale.

Per quanto riguarda l'intelligibilità della terminologia medica, essa è in gran parte determinata dalle conoscenze della scienza medica. Il punto discorante tra il polacco e l'italiano medico è però costituito dalle nozioni tecniche possedute, necessarie per una comprensione approssimativa da parte del parlante nativo non specialista. Sebbene la terminologia medica né in polacco né in italiano appartenga al lessico ad alto uso, la base neoclassica fino ad un certo punto può contribuire ad una maggiore comprensione del termine da parte degli italofoni, visto che:

[...] la maggior parte dell'italiano, composto secondo il dizionario De Mauro da 2071 parole, sono di origine latina 1710 (l' $82 \%)$; e percentuali simili emergono anche per le parole ad alto uso (2663, di cui di origine latina 1858, ossia il 69\%). Nel lessico ad alto uso è cospicuo il numero dei vocaboli che non solo sono derivati dal latino, ma che sono anche latini, nel senso che li troviamo identici nel vocabolario latino, e se differiscono dalle corrispondenti voci italiane è solo per piccoli adattamenti morfologici o grafici [...]. (Poli 18).

Nel caso del linguaggio medico polacco, perfino la base etimologica dei termini specialistici si distacca dalla lingua usata quotidianamente, per cui al processo di comprensione non giovano né conoscenze linguistiche né tanto meno quelle cognitive. Si ritiene che la presenza dei composti neoclassici nella lingua polacca, nonché l'introduzione di nuovi termini non comportino automaticamente la loro comprensione da parte dei parlanti nativi: 
[...] masowe powielanie takich form nie świadczy jeszcze o tym, by były one właściwie rozumiane przez użytkowników. Zależy to od ogólnego wykształcenia i znajomości języków obcych, a także od wiedzy na temat historii własnego języka, skłonności do etymologizowania itd. Tak więc w dużym stopniu subiektywne są same granice podzielności i przejrzystości konstrukcji ${ }^{10}$ (Nagórko 203).

Data questa maggiore esclusività della forma lessicale dei termini specialistici, il loro intendimento è riservato prevalentemente agli specialisti abituati forse maggiormente al loro uso sia per le loro solide basi cognitive che per l'esposizione quotidiana a termini internazionali riscontrati in pubblicazioni scientifiche.

L'origine della doppia terminologia polacca è spiegata parzialmente dal prestigio del latino e dal greco che sotto forma di prestiti sono stati introdotti nel vocabolario medico:

[...] zapożyczone z greki termin naukowe, które na przełomie XIX i XX wieku były nieakceptowane przez normę, funkcjonują współcześnie z jednej strony ze względu na to, że nie miały rodzimych odpowiedników, którymi można było je zastąpić, a z drugiej dlatego, że polskie synonimy nie były wystarczająco precyzyjne. W związku z tym nie można było traktować ich jako terminów o charakterze specjalistycznym. Proponowane odpowiedniki miały zwykle więcej niż jedno znaczenie, zaś pożyczki greckie odnosiły się wyłącznie do sfery nauki (szczególnie medycyny). (Kupidura 205)

I traduttori del linguaggio medico in polacco, memori della genesi della terminologia medica, saranno più propensi a seguitare questa tradizione lessicale. Per i testi di carattere divulgativo (foglietti illustrativi, consenso informato ecc.) riserveranno i termini di origine slava, mentre i loro analoghi neoclassici verranno usati nei testi altamente specialistici (articoli scientifici, scrittura professionale tra medici, reparti ecc.). Tale distinzione è però solo una proposta a titolo orientativo, in quanto ogni testo costituisce un caso a sé stante raramente soggetto all'interpretazione basata sul ragionamento algoritmico.

10 "Una ripetizione massiccia di tali forme non dimostra ancora che siano adeguatamente comprese dai parlanti. Dipende dall'istruzione generale e dalla conoscenza delle lingue straniere, nonché dalla conoscenza della storia della propria lingua e dalla consapevolezza etimologica ecc. Pertanto, i limiti stessi della divisibilità e della trasparenza di queste strutture sono in larga misura soggettivi".

11 “[...] termini scientifici presi in prestito dal greco, che a cavallo tra il XIX e il XX secolo erano inaccettabili per la norma, sono attualmente in uso da un lato perché non avevano equivalenti nativi con cui poter essere sostituiti, e dall'altro perché i sinonimi polacchi non erano abbastanza precisi. Pertanto, non potevano essere trattati come termini specialistici. Le versioni proposte di solito avevano più di un significato, mentre i prestiti greci si riferivano esclusivamente al regno della scienza (specialmente della medicina)". 


\section{PAROLE POLIREMATICHE}

Prima di illustrare la natura delle parole politematiche in chiave comparativa è opportuno ricordare i loro criteri distintivi. Sia in polacco che in italiano le parole polirematiche (in polacco definite come zestawienia) sono parole di due o più elementi, principale caratteristica delle quali consiste nell'ordine fisso delle loro singole parti: il termine è completo se e solo se è costituito nell'ordine prestabilito da elementi che compongono un senso figurato desumibile dall'insieme dei suoi elementi. Secondo la definizione delle polirematiche italiane proposta da Grossmann e Rainer: "[...] le polirematiche sono considerate l'esito di processi di lessicalizzazione, quindi hanno un significato non composizionale, cioè non desumibile dalla somma dei significati delle parole che le compongono; il loro significato pertanto può essere o figurato oppure non iponimo della testa" (36).

La definizione delle polirematiche sfugge però ad una classificazione univoca, in quanto gli stessi conoscitori dell'argomento non sono d'accordo sulla natura esatta del fenomeno, affermando da una parte che "le formazioni che manifestano tra il complesso e la sua testa una relazione di iponimia/ iperonimia e il cui significato è composizionale possono essere definite composti mentre saranno classificabili tra le polirematiche le formazioni che non manifestano tali proprietà" (Grossmann, Rainer 36). Dall'altra parte però si afferma che ogni singola unità lessicale facente parte di una parola polirematica in grado variabile rinvia al proprio significato originario. Il senso unico della polirematica viene quindi costruito dall'accostamento di sensi molteplici: “[...] la natura stessa dell'unità polirematica concorre molto spesso alla mancanza di un trattamento univoco; per cui alcuni tipi di costrutti si prestano meglio di altri ad un'entrata autonoma e altri, per converso, indirizzano verso la propria sistemazione all'interno di un'altra voce" (De Fazio 135).

Per quanto riguarda le parole polirematiche classificate in polacco come zestawienie, esse si distinguono per la forma fissa di due o più parole che le compongono (Urbańczyk 401; Bąk 228, Jadacka 120), per esempio:

Tab. 4. Raffronto di parole polirematiche italiane e polacche

\begin{tabular}{|c|c|}
\hline termini medici italiani & termini medici polacchi \\
\hline aorta toracica & aorta piersiowa \\
\hline addensamento parenchimale & zagęszczenie miąższowe \\
\hline
\end{tabular}




\begin{tabular}{|c|c|}
\hline ansa intestinale & pętla jelitowa \\
\hline antro gastrico & oddźwiernik żołądka \\
\hline cicatrice chirurgica & blizna chirurgiczna \\
\hline cisti sebacea & torbiel łojowa \\
\hline lesioni focali & zmiany ogniskowe \\
\hline lesione solida & zmiana lita \\
\hline lingua bifida & rozszczep języka/rozdwojenie języka \\
\hline liquido peritoneale & płyn opłucnowy \\
\hline filtrato glomerulare & przesączanie kłębuszkowe \\
\hline forame di congiunzione & otwór międzykręgowy \\
\hline fornice vaginale & sklepienie pochwy \\
\hline murmure vescicolare & szmer oddechowy pęcherzykowy \\
\hline ombra cardiaca & sylwetka serca \\
\hline osteite fibrocistica & dystrofia kości przytarczyczkowa \\
\hline pancreatite emorragica acuta & zapalenie trzustki ostre krwotoczne \\
\hline papilla minor & brodawka dwunastnicy mniejsza \\
\hline polso pedido & tętno w tętnicy grzbietowej \\
\hline processo spinoso & wyrostek kolczysty kręgu \\
\hline soffio vascolare & szmer naczyniowy \\
\hline tessuto sottocutaneo & tkanka podskórna \\
\hline toni parafonici & tony głuche \\
\hline vena porta & żyła główna \\
\hline versamento libero & wysięk wolny \\
\hline vertebra farfalla & kręg motyli \\
\hline vie biliari & drogi żółciowe \\
\hline
\end{tabular}


La lista di parole polirematiche italiane include prevalentemente termini composti da nome e aggettivo, più raramente da nome e un complemento, mentre in polacco capita che alcuni termini vengano sviluppati per aggiunta di un terzo o quarto elemento. Ciò potrebbe essere dovuto parzialmente al fatto che nella terminologia polacca più spesso si manifestano casi di polirematiche e non di composti. Nel caso dell'italiano, invece, una maggiore densità di significato è rinchiusa nel composto, il quale, se viene a trovarsi in un'unità polirematica, non aggiunge ulteriori elementi lessicali, per esempio: osteite fibrocistica $>$ dystrofia kości przytarczyczkowa; pancreatite emorragica acuta $>$ zapalenie trzustki ostre krwotoczne.

Può succedere che il traduttore dall'italiano incorra in termini che potrebbero essere interpretati come parole polirematiche, ossia come termini dal senso deducibile e completo solo in compresenza di tutti gli elementi dell'espressione. Servirà perciò rammentare anche il criterio stilistico del linguaggio medico italiano e non solo quello terminologico. Infatti, l'italiano medico tende a creare termini apparentemente specialistici, la cui forma più semplificata circola nell'uso comune della lingua, per esempio: paziente apiretico = paziente senza febbre, frattura costale = frattura della costa; non espansi di pertinenza surrenalica $=$ surrene non espanso. La distinzione del ruolo dell'aggettivo di relazione in questo tipo di termine non sempre è del tutto ovvia, basti citare l'esempio di formazione espansiva. Il termine come tecnicismo significa una struttura che tende all'accrescimento, ma che non può essere denominata altrimenti prima che vengano eseguiti ulteriori accertamenti.

Un'altra avvertenza nel caso dell'interpretazione delle polirematiche consiste nel fatto che in italiano, nella scrittura professionale si tende ad eliminare uno o più elementi, perciò non è affatto raro riscontrare le espressioni come porta pervia, l'ombra ingrandita. Una certa incoerenza nella citazione delle polirematiche si può manifestare perfino in diversi manuali destinati all'uso dell'italiano medico:

Anche nell'organizzazione delle parole polirematiche non vi è molta trasparenza: alcune voci presentano il solo nome senza premettere il sostantivo di riferimento: dentato anteriore (muscolo), estensore lungo delle dita (muscolo), ecc. in altri casi, invece, per disambiguare, quest'ultimo è premesso, ad esempio muscoli facciali, muscoli papillari, in altri infine, è presente ma posposto surrenale (ghiandola). (Polita 355-356).

Pare che l'eliminazione di un elemento dalle polirematiche dipenda da alcuni fattori, tra cui si possono indicare: 
a. l'espressione si è ormai affermata nella sua forma parziale, per cui pare superfluo riportare il termine completo, per esempio: (vena) porta, limitante (vertebrale).

b. il secondo elemento è solo una specificazione di un insieme maggiore, per esempio: (muscolo) deltoide, (vena) succlavia, (aorta) coronaria.

c. il co- e contesto sono tanto ampi da agevolare una comprensione inequivocabile di singoli termini, per esempio: testa (del pancreas) appare globosa; (test di) Romberg negativo.

Qualora l'eliminazione di un elemento dalla polirematica possa essere causa di incomprensione, il termine viene conservato integro, per esempio: angina da sforzo, blocco di Branca. Pare che alcune polirematiche si adattino meglio a questi procedimenti di riduzione, in particolare quando con l'elisione di un elemento viene conservato il legame logico e la relazione di ipoo iperonimia. Per esempio in un esame ecografico del pancreas o dell'utero il medico può benissimo usare solo un termine parziale testa (del pancreas), o collo (dell'utero), siccome è l'intero testo a contestualizzare la parte del termine mancante. In un altro caso, con l'eliminazione della testa della polirematica un secondo elemento rimanente nella relazione di iponimia per composizione viene trasformato in un altro sostantivo, per esempio: il (muscolo) deltoide, la (vena) safena.

Dal punto di vista del traduttore, il processo di composizione ${ }^{12}$ frequentissimo nella terminologia medica potrà portare alla creazione di termini polisemici, la comprensione dei quali non sempre deve essere facilitata dal contesto, per esempio: la poplitea, l'ascellare, la succlavia, la brachiale possono riferirsi sia alla vena che all'arteria. Nel caso della lingua polacca, almeno nel linguaggio scritto, questo fenomeno pare meno frequente. Ciò potrebbe essere spiegato col fatto che diversamente dall'italiano, nel polacco sono molto meno frequenti derivati omofoni ed omografi, a preferenza di formazioni con affissi (Kaproń-Charzańska 105-120).

In questa breve rassegna di parole composte e polirematiche dall'ambito medico si è cercato di indicare alcuni punti critici che il traduttore potrà riscontrare nei testi medici polacchi ed italiani. Si è voluto mettere l'accento su alcune difficoltà terminologiche che possono sorgere nel momento di raffronto delle lingue durante il processo di traduzione e che non sempre

\footnotetext{
${ }^{12}$ Senza addentrarci nell'argomento, si segnala solo che alcuni studiosi invece di composizione parlano di derivazione/suffissazione zero, ritenendo che la formazione del derivato avvenga per aggiunta di appunto un affisso zero (Don).
} 
possono essere facilmente risolte. Le due lingue, con la loro lunghissima storia, sono sorprendentemente molto simili per quanto concerne il linguaggio medico e a maggior ragione il traduttore nella sua prassi deve stare all'erta, affinché la terminologia medica di una lingua non straripi, inondando con i suoi tratti distintivi l'altro sistema linguistico.

\section{BIBLIOGRAFIA}

Arcodia, Giorgio. La derivazione lessicale in cinese mandarino. FrancoAngeli, 2008.

Bąk, Piotr. Gramatyka języka polskiego. Wiedza Powszechna, 1989.

Berruto, Gaetano. Sociolinguistica dell'italiano contemporaneo. La Nuova Italia Scientifica, 1987.

Cabitza, Paolo. Ortopedia. Società Editrice Esculapio, 2013.

Canepari, Michela. Lingua e traduzione, vol. I. I fondamenti. Libreria Universitaria, 2016.

Dardano, Maurizio. La formazione delle parole nell'italiano di oggi (primi materiali e proposte). Bulzoni, 1978.

De Fazio, Debora. "Il trattamento delle unità polirematiche nel Dizionario della Lingua Italiana di Tommaso-Bellini”. XXVI Congreso Internacional de Lingüistica y de Filología Románicas, eds. Casanova Herrero Emili, Calvo Rigual Cesáreo, de Gruyter, 2013, pp. 135-145.

De Mauro, Tullio. La fabbrica delle parole: il lessico e problemi di lessicologia. UTET, 2005.

Don, Jan. Morphological conversion. Led, 1993.

Garzone, Giuliana, Francesca Santulli. Il linguaggio giuridico. Prospettive interdisciplinari. Giuffrè Editore, 2008.

Głowiński, Michał. Słownik terminów literackich. Zakład Narodowy im. Ossolińskich, 2010.

Grochola-Szczepanek, Helena. "Główne typy strukturalno-semantyczne rzeczowników złożonych w dialektach polskich”, Jezikoslovni Zapiski n. 14, 2008, pp. 61-74.

Grossmann, Maria, Franz Rainer. La formazione delle parole in italiano. Max Niemeyer Verlag, 2004.

Handke, Kwiryna. Budowa morfologiczna i funkcje compositów polskich: z uwzględnieniem innych języków zachodniosłowiańskich. Zakład Narodowy im. Ossolińskich, 1976.

Holtus, Günter, Michael Metzeltin, Christian Schmitt. Lexikon der Romanistischen Linguistik. Max Niemeyer Verlag, 1988.

Ibba, Maria. L'inglese della medicina. Vita e pensiero,1988.

Jadacka, Hanna. Kultura języka polskiego. PWN, 2013.

Kaproń-Charzańska, Iwona. "O nowych odczasownikowych i odrzeczownikowych nazw subiektów", Polonica n. 28, 2007, pp. 105-120.

Krucka, Barbara. "Związki frazeologiczne $\mathrm{z}$ czasownikiem robić i ich ekwiwalenty w języku rosyjskim", Acta Universitatis Lodziensis, n. 6, 1996, pp. 23-34.

Kupidura, Daria. "Lacińskie i greckie nienormatywne zapożyczenia w polszczyźnie XIX wieku a ich żywot w polszczyźnie drugiej połowy XX wieku", Synchroniczne i diachroniczne aspekty badań polszczyzny n. 15-13, 2016, pp. 195-210. 
Laghi, Andrea, Marco Rengo. Protocolli in TC spirale multistrato. Vol. 3 Cuore-torace. Springer Verlag Italia, 2009.

Lalla, Claudio. Medicina naturale per prevenire il cancro. Franco Angeli, 2009.

Montermini, Fabio. ll lato sinistro della morfologia. La prefissazione in italiano e nelle lingue del mondo. Franco Angeli, 2008.

Nagórko, Alicja. Podręczna gramatyka języka polskiego. Wydawnictwo Naukowe PWN, 2010.

Poli, Andrea. Le "due" culture e il latino. Fra linguistica, letteratura, filosofia, psicologia cognitiva ed epistemologia. Youcanprint, 2015.

Polita, Maria. "L'apprendimento della lingua della medicina in L2: due manuali a confronto", Italiano Lingua Due, n. 2, 2013, p. 341-365.

Serianni, Luca. Un treno di sinonimi. I medici e le parole: percorsi linguistici nel passato e nel presente. Garzanti, 2005.

Tekavčić, Pavao. Grammatica storica dell'italiano, III. Lessico. Il Mulino, 1980.

Urbańczyk, Stanisław. Encyklopedia wiedzy o języku polskim. Zakład Narodowy im. Ossolińskich, 1978.

Zieliński, Krzysztof. Stownik pochodzenia nazw i określeń medycznych. Antyczne i nowożytne dzieje chorób w ich nazwach ukryte. Alfa Medica Press, 2004.

\section{STUDIO COMPARATIVO DELLE PAROLE COMPOSTE E POLIREMATICHE NEL LINGUAGGIO MEDICO ITALIANO E POLACCO}

\section{Riassunto}

Dardano ricorda che nella terminologia medica italiana "la componente latina e greca costituisce un patrimonio cospicuo" (Dardano 501, 514). Lo stesso si può dire per il polacco, sebbene gli elementi greco-latini usati nel linguaggio medico siano molto spesso accompagnati dai loro sinonimi di origine slava. Mentre in italiano i termini come condromatosi, faringoplastica o nefropatia fanno parte sia del linguaggio tecnico che divulgativo, in polacco si osservano coppie terminologiche come: chondromatoza - chrzęstniakowatość, faryngoplastyka - plastyka gardła, nefropatia - choroba nerek, usate a seconda della situazione comunicativa e del registro.

In italiano, in molti casi, è possibile formare sintagmi preposizionali e aggettivali come: ernia del disco/ernia discale, exeresi dei linfonodi/exeresi linfonodale. La costruzione con il complemento di specificazione, sebbene ammissibile in certi casi, non sembra adeguata al registro alto del linguaggio specialistico. I termini corrispondenti in polacco avranno invece una sola variante con il complemento di specificazione: przepuklina kręgostupa, usunięcie węzłów chłonnych.

Lo studio comparativo delle parole complesse e polirematiche esistenti nella terminologia medica polacca e italiana permetterà di individuare eventuali differenze nei due sistemi linguistici e indicarne le funzioni sintattico-semantiche.

Parole chiave: language for specific purpose; medicina; composti; polirematiche. 


\title{
ANALIZA PORÓWNAWCZA ZŁOŻEŃ I ZESTAWIEŃ W POLSKIM I WŁOSKIM JEZYYKU MEDYCZNYM
}

\author{
Streszczenie
}

Dardano przypomina, że we włoskiej terminologii medycznej „elementy łacińskie i greckie stanowią widoczne dziedzictwo" (Dardano 501, 514). To samo można powiedzieć o polszczyźnie, choć elementom grecko-łacińskich obecnym w języku medycznym bardzo często towarzyszą synonimy pochodzenia słowiańskiego. O ile w terminologii włoskiej chondromatoza, faryngoplastyka czy nefropatia należą zarówno do języka specjalistycznego, jak i potocznego, o tyle w języku polskim istnieją pary terminologiczne, takie jak: chondromatoza - chrzęstniakowatość, faryngoplastyka plastyka gardła, nefropatia - choroba nerek, używane w zależności od sytuacji komunikacyjnej oraz rejestru.

W języku włoskim w wielu przypadkach możliwe jest tworzenie syntagm przyimkowych i przymiotnikowych, na przykład: ernia del disco/ernia discale, exeresi dei linfonodi/exeresi linfonodale. Terminy specjalistyczne $\mathrm{z}$ dopełnieniem, choć są dopuszczalne, nie wydają się adekwatne w rejestrze wysokim języka specjalistycznego. Odpowiednie określenia w języku polskim będą miały tylko jeden wariant: przepuklina kręgosłupa, usunięcie węzłów chłonnych.

Analiza porównawcza złożeń i zestawień występujących w polskiej i włoskiej terminologii medycznej pozwoli wskazać na różnice w obu systemach językowych oraz ich funkcje syntaktyczno-semantyczne.

Słowa kluczowe: języki specjalistyczne; medycyna; złożenia; zestawienia. 